\title{
Lifting Preferences over Alternatives to Preferences over Sets of Alternatives: The Complexity of Recognizing Desirable Families of Sets
}

\author{
Jan Maly \\ Institute of Logic and Computation, \\ TU Wien, Austria \\ jmaly@dbai.tuwien.ac.at
}

\begin{abstract}
The problem of lifting a preference order on a set of objects to a preference order on a family of subsets of this set is a fundamental problem with a wide variety of applications in AI. The process is often guided by axioms postulating properties the lifted order should have. Well-known impossibility results by Kannai and Peleg and by Barberà and Pattanaik tell us that some desirable axioms - namely dominance and (strict) independence - are not jointly satisfiable for any linear order on the objects if all non-empty sets of objects are to be ordered. On the other hand, if not all non-empty sets of objects are to be ordered, the axioms are jointly satisfiable for all linear orders on the objects for some families of sets. Such families are very important for applications as they allow for the use of lifted orders, for example, in combinatorial voting. In this paper, we determine the computational complexity of recognizing such families. We show that it is $\Pi_{2}^{p}$-complete to decide for a given family of subsets whether dominance and independence or dominance and strict independence are jointly satisfiable for all linear orders on the objects if the lifted order needs to be total. Furthermore, we show that the problem remains coNP-complete if the lifted order can be incomplete. Additionally, we show that the complexity of these problem can increase exponentially if the family of sets is not given explicitly but via a succinct domain restriction.
\end{abstract}

\section{Introduction}

Modeling preferences over alternatives is a major challenge in many areas of AI, for example in knowledge representation and, especially, in computational social choice. If the number of alternatives is small enough, preferences are most often modeled as a total order. However, in many applications the alternatives are 'combinatorial', for example bundles of objects in packing or allocation problems (Bouveret, Chevaleyre, and Maudet 2016), or committees in voting (Brandt and Brill 2011; Lang and Xia 2016; Brandt, Saile, and Stricker 2018). In such situations, the number of alternatives grows exponentially with the number of objects which makes it unfeasible for agents to specify a full preference relation over all alternatives.

Copyright (c) 2020, Association for the Advancement of Artificial Intelligence (www.aaai.org). All rights reserved.

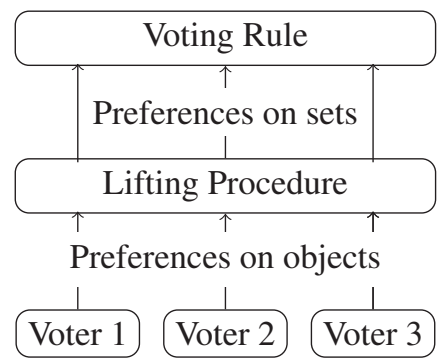

Figure 1: The use of lifted orders in voting

Different approaches to solving this problem have been discussed in the context of voting. Lang and Xia (2016) give a thorough overview of the most promising ideas. The easiest solution is often to vote on each candidate separately. However, this approach only works well if the voters have separable preferences, i.e. if the preference on having a candidate in the committee is independent on who else is in the committee. Another option is eliciting the top ranked committee. Then one can, for example, infer a preference order on the committees via a distance measure like the Hamming distance. This approach minimizes the communication cost but only takes very little of the agent's full preferences into account. Alternatively, one can ask the agents to specify their preferences using a CP-net or similar representations. This can be very effective but requires the agents to learn a nontrivial preference representation. In many cases this is an unacceptable requirement. Finally, there are some voting rules that select a winning committee directly from a preferences over candidates. This approach has been mainly explored for committees of fixed size. In this paper we consider a different approach, namely inferring an order on sets of alternatives from an order on the alternatives. Then, traditional voting rules can be applied to the inferred orders on sets. Figure 1 shows how this approach can work in voting. Barberà, Bossert, and Pattanaik (2004) give an excellent survey on the progress that has been made to solve this question.

Essentially, there are two ways to study this approach. First, one can analyze specific methods to infer an order on sets from an order on the objects, see e.g. (Moretti and 
Tsoukiàs 2012). Second, one can analyze which properties an optimal order on the sets should have in a given setting. These desirable properties can then be formulated as axioms and one can try to identify orders that satisfy these axioms. In some settings, it is possible to find orders that satisfy all properties deemed desirable in the setting, see e.g. (Pattanaik and $\mathrm{Xu}$ 1990). Unfortunately, in many other settings no order can satisfy all desirable properties at once. One of the most important of these so-called impossibility results states that two axioms called Dominance and Independence are in general incompatible (Kannai and Peleg 1984). Dominance states, intuitively, that removing the least preferred element from a set improves the set and removing the most preferred element worsens the set. Independence states, roughly, that if a set is preferred to another set and the same element is added to both sets, this preference can not be reversed.

Kannai and Peleg (1984) showed that dominance and independence are incompatible if one wants to order all subsets of a set with at least six elements. In the same year Barberà and Pattanaik (1984) showed that dominance and a strengthening of independence called strict independence are incompatible already if one wants to order all subsets of a three element set. We write '(strict) independence' as a shorthand for 'independence or strict independence'. Recent research focused on new impossibility results, e.g. Geist and Endriss (2011), as well as weakenings and variations of dominance and independence, e.g. Bossert and Suzumura (2000) and Larbi, Konieczny, and Marquis (2010).

All of the results above assume that all subsets of a given set need to be ordered, but in many applications only some of these subsets are possible alternatives. For example, it is very common in multiwinner or combinatorial voting to have some form of domain restrictions (Lang and Xia 2016; Kilgour 2016). Now, it is possible to construct arbitrary large families of sets - for example families of disjoint sets that can be ordered with an order satisfying dominance and (strict) independence. Motivated by this observation, Maly and Woltran (2017) have shown that it is NP-complete to decide whether a given order on elements can be lifted to an order satisfying dominance and (strict) independence.

However, for applications in voting or other social choice problems, it is necessary to fix a voting method before the ballots are collected. Therefore, it is more important to know for a given family of sets if dominance and (strict) independence are compatible for any preference order the agents may report. ${ }^{1}$ Following Maly, Truszczyński, and Woltran (2018) we call families of sets for which any possible order on the elements can be lifted to an order satisfying dominance and (strict) independence strongly orderable with respect to dominance and (strict) independence. Maly, Truszczyński, and Woltran (2018) studied this concept for a specific class of families of sets, namely for families of sets that can be represented as the family of all sets of vertices

\footnotetext{
${ }^{1}$ Observe that the hardness of this problem does not follow from the hardness of the aforementioned problem treated by Maly and Woltran (2017). For example, the question whether a given boolean formula over a set of variables is satisfiable is NPcomplete, but the question whether all or at least one boolean formula over a set of variables is satisfiable is trivial.
}

that induce a connected subgraphs in a given graph. One of their main results is a classification result that implies that strong orderability with respect to dominance and strict independence can be decided in polynomial time for families in this restricted class.

In this paper, we show that this result can not be generalized to arbitrary families of sets. We show that it is in general $\Pi_{2}^{p}$-complete to decide whether a family of sets is strongly orderable with respect to dominance and strict independence. The result also holds if we replace strict independence by independence. These results assume that we require the order on the family of sets to be total. However, some authors argue that it is more sensible to only require incomplete preferences in combinatorial domains (Boutilier and Rosenschein 2016). Voting rules that facilitate the aggregation of partial orders or even weaker preference models exist (Xia and Conitzer 2011; Terzopoulou and Endriss 2019). Therefore, we investigate how much the complexity of the studied problems can be reduced by dropping the requirement that the lifted order needs to be total. In particular, we show that it is coNP-complete to decide whether a family of sets is strongly orderable if we require the order on the family to be a partial order.

These results assume that the family of sets is given explicitly. However, the domain in combinatorial voting is of ten given as a condition that has to be satisfied by the admissible sets. Such conditions can for example be formulated as propositional formulas (Lang and Xia 2016). These formulas are normally exponentially smaller than the actual family of sets, which can increase the complexity of deciding if the family is strongly orderable. On the other hand, families of sets must have some internal structure to be succinctly represented. This internal structure may decrease the complexity of the problem, as is the case for the domain restrictions considered by Maly, Truszczyński, and Woltran (2018). We show - for a specific succinct representation that is well studied in the literature - that succinct representation can lead to a massive blow up in complexity. It turns out that it can be NEXP-hard to decide whether a succinctly represented family is strongly orderable with respect to dominance and strict independence for total orders and coNEXPcomplete for partial orders. The first result also holds if only independence is required instead of strict independence.

After formally introducing our research questions in the next section, we will present our main contributions in the third section. These are:

1. We show that it is $\Pi_{2}^{p}$-complete to decide whether a family of sets is strongly orderable with respect to dominance and (strict) independence. This result implies also that it is not possible to find an order satisfying dominance and (strict) independence in polynomial time even if one already knows that a given family is strongly orderable.

2. For dominance and strict independence, we prove that it is still coNP-complete to decide if a family of sets is strongly orderable if one only requires a partial order on the family of sets.

3. We show that the we can have an exponential blow up in complexity if the family of sets is represented succinctly. 
Using a so-called conversion lemma, we show that our hardness results imply NEXP- resp. coNEXP-hardness for a specific succinct representation.

\section{Background}

First, we recall some general background. All sets we consider in the paper are finite. A binary relation is called a preorder if it is reflexive and transitive. A preorder that is antisymmetric is called a partial order. A preorder that is total is called a weak order. A weak order is linear if it is also antisymmetric. If $\preceq$ is an order on a set $X$, then the corresponding strict order $\preceq$ on $X$ is defined by $x \prec y$ if $x \preceq y$ and $y \npreceq x$, where $x, y$ are arbitrary elements of $X$. Further, the corresponding equivalence or indifference relation $\sim$ is defined by $x \sim y$ if $x \preceq y$ and $y \preceq x$. For a linear order $\preceq$ on a set $A$, we write $\max _{\preceq}(A)$ for the maximal element of $A$ with respect to $\preceq$. Similarly, we write $\min \preceq(A)$ for the minimal element of $A$ with respect to $\preceq$. If no ambiguity arises, we omit the $\preceq$ in the subscript.

We assume that the reader is familiar with the classes NP and coNP. We will write SAT for the NP-complete problem of deciding if a 3-CNF is satisfiable and TAUT for the coNP-complete problem of deciding if a 3-DNF is a tautology. We additionally use the class NEXP of problems solvable by a nondeterministic Turing Machine in exponential time, its complement class coNEXP and $\Pi_{2}^{p}$, the class of problems that are in coNP using an NP-oracle. An example for a $\Pi_{2}^{p}$-complete problem is $\Pi_{2}$-SAT i.e. the problem of deciding whether a quantified boolean formula of the form $\forall \vec{W} \exists \vec{V} \psi(\vec{W}, \vec{V})$, where $\psi$ is a formula in 3-CNF, is satisfiable. For more details the reader is referred to the famous textbook by Papadimitriou (1994).

Next, we review the relevant background on lifting orders from objects to sets of objects. Formally, we model this problem using a set $X$, a family $\mathcal{X} \subseteq \mathcal{P}(X) \backslash\{\emptyset\}$ of non-empty subsets of $X$ and a linear order $\leq$ on $X$. Then, we want to infer from $\leq$ an order $\preceq$ on $\mathcal{X}$, guided by axioms formalizing certain desiderata for such lifted orders. We call this the order lifting problem. We recall the axioms that are relevant for this paper, i.e. dominance, independence and strict independence. They are natural extensions of the versions of those axioms considered in the case when $\mathcal{X}=\mathcal{P}(X) \backslash\{\emptyset\}$. The extensions consist of adding conditions of the form $Y \in \mathcal{X}$ not needed in the original formulations (Maly and Woltran 2017).

Axiom 1 (Dominance). For all $A \in \mathcal{X}$ and all $x \in X$, such that $A \cup\{x\} \in \mathcal{X}$ :

$$
\begin{aligned}
& y<x \text { for all } y \in A \text { implies } A \prec A \cup\{x\}, \\
& x<y \text { for all } y \in A \text { implies } A \cup\{x\} \prec A .
\end{aligned}
$$

Axiom 2 (Independence). For all $A, B \in \mathcal{X}$ and for all $x \in X \backslash(A \cup B)$, such that $A \cup\{x\}, B \cup\{x\} \in \mathcal{X}$ :

$$
A \prec B \text { implies } A \cup\{x\} \preceq B \cup\{x\} \text {. }
$$

Axiom 3 (Strict Independence). For all $A, B \in \mathcal{X}$ and for all $x \in X \backslash(A \cup B)$, such that $A \cup\{x\}, B \cup\{x\} \in \mathcal{X}$ :

$$
A \prec B \text { implies } A \cup\{x\} \prec B \cup\{x\} \text {. }
$$

Dominance is often desirable if the order $\preceq$ should reflect, to some extent, the average quality of a set or committee. If we assume, for example, that the sets represent incompatible alternatives from which one will be chosen randomly, then dominance is a natural desideratum (Can, Erdamar, and Sanver 2009). Independence and strict independence on the other hand are simple monotonicity axioms. They are sensible desiderata in many interpretations, for example if sets are bundles of objects that are compared according to their overall goodness according to some additive utility (Kraft, Pratt, and Seidenberg 1959).

There is some tension between the motivations for dominance and (strict) independence, as dominance is more related to average utility while independence and strict independence are more related to total utility. Nevertheless, there are important settings where both axioms are natural desiderata. One example for such a situation is choice under complete uncertainty (Bossert, Pattanaik, and Xu 2000; Barberà, Bossert, and Pattanaik 2004), i.e. the situation that the set represents mutually exclusive alternatives that are chosen with a unknown probability. In combinatorial voting comparable situations occur, for example, when the agents are unaware of the influence of any given candidate in the elected committee.

Example 1. Let $X=\{a, b, c\}$ be a set of candidates from which a committee should be elected. Assume further, that any winning committee must contain either a or $b$. Then, the family of possible winning committees $\mathcal{C}$ contains all possible committees except $\{c\}$. Now assume one voter reports the preference order $a<b<c$. Then these preferences can be lifted to a linear order on $\mathcal{C}$ that satisfies dominance and strict independence. For example:

$$
\{a\} \prec\{a, b\} \prec\{b\} \prec\{a, b, c\} \prec\{a, c\} \prec\{b, c\}
$$

Now, if an agent reports the preference order $a<c<b$, then no linear order on $\mathcal{C}$ satisfies dominance and strict independence with respect to the agents preferences. However, if we replace strict independence by independence and do not require the order to be strict, then for example

$$
\{a\} \prec\{a, b\} \prec\{a, b, c\} \sim\{a, c\} \prec\{b, c\} \prec\{c\}
$$

satisfies dominance and independence with respect to the agents preferences.

We observe that dominance and strict independence only mention strict preferences. Therefore, we can expect the order $\preceq$ to additionally be strict if we consider dominance and strict independence. This extra requirement is natural in computational social choice as most voting rules assume strict orders as input. Furthermore, it does simplify some of the proofs. However, is not necessary for the results to hold. If we consider dominance and independence, we only assume that $\preceq$ is a (pre-)order, as independence coincides with strict independence on strict orders. We use the following notation to simplify presentation.

Definition 2. Let $X$ be a set and $\mathcal{X} \subseteq \mathcal{P}(X) \backslash\{\emptyset\}$. We say $\mathcal{X}$ is $D I$-orderable with respect to a linear order $\leq$ on $X$ if there exists a weak order $\preceq$ on $\mathcal{X}$ satisfying dominance and independence with respect to $\leq$. 


\begin{tabular}{lll}
\hline Orderability & Dom + Ind & Dom + strict Ind \\
\hline partial ord. w.r.t. $\leq$ & always $^{\dagger}$ & in $^{\dagger}$ \\
partial strong ord. & always & coNP-c. \\
ord. w.r.t. $\leq$ & NP-c. & NP-c. \\
strong ord. & $\Pi_{2}^{p}$-c. & $\Pi_{2}^{p}$-c. \\
succ. part. strong ord. & coNEXP-c. & coNEXP-c. \\
succinct ord. w.r.t. $\leq$ & NEXP-c. & NEXP-c. \\
succinct strong ord. & NEXP-hard & NEXP-hard \\
\hline
\end{tabular}

Table 1: Complexity of orderability with respect to dominance and (strict) independence.

We say $\mathcal{X}$ is $D I^{S}$-orderable with respect to a linear order $\leq$ on $X$ if there exists a linear order $\preceq$ on $\mathcal{X}$ satisfying dominance and strict independence with respect to $\leq$.

Observe that for $\mathcal{X} \neq \mathcal{P}(X) \backslash\{\emptyset\}$, dominance and (strict) independence do not imply the extension rule, which is a minimal requirement on lifted orders in many applications.

Axiom 4 (The extension rule). For all $x, y \in X$, such that $\{x\},\{y\} \in \mathcal{X}$, if $x<y$ then $\{x\} \prec\{y\}$.

However, adding the extension rule has no effect on complexity (see Remark 15). To be able to talk about complexity we have to consider the corresponding decision problems.

\section{$D I^{S}$-ORDERABILITY}

Input: $\quad$ A set $X$, a family of sets $\mathcal{X} \subseteq \mathcal{P}(X) \backslash\{\emptyset\}$ and a linear order $\leq$ on $X$.

Question: Is $\mathcal{X} D I^{S}$-orderable with respect to $\leq$ ?

$D I$-ORDERABILITY is defined analogously by replacing $D I^{S}$-orderable with $D I$-orderable. Maly and Woltran (2017) showed that $D I$-ORDERABILITY and $D I^{S_{-}}$ ORDERABILITY are NP-complete.

The main focus of this paper is not $D I$ - or $D I^{S_{-}}$ orderability with respect to a specific linear order $\leq$ on $X$ but with respect to every linear order on $X$.

Definition 3. We say $\mathcal{X}$ is strongly $D I-$ resp. $D I^{S}$-orderable if it is DI-resp. DI $I^{S}$-orderable with respect to every linear order on $X$.

Using this notation, we can say that the family considered in Example 1 is $D I^{S}$-orderable with respect to the order $a<$ $b<c$. It is not $D I^{S}$-orderable with respect to the order $a<c<b$ though and therefore not strongly $D I^{S}$-orderable. On the other hand, the family is $D I$-orderable with respect to the order $a<c<b$ and it is easy to check that it is even strongly $D I$-orderable.

\section{Results}

In this section we will cover our results. First, we consider the problem of determining if a set is strongly $D I$ or $D I^{S_{-}}$ orderable. Then, we will investigate how the complexity of these problems is affected if we drop the requirement that the lifted order needs to be total. Finally, we will look at the effect of succinct domain restrictions on complexity. An overview over all our main results is given in Table 1

\footnotetext{
${ }^{\dagger}$ (Maly and Woltran 2017)
}

\section{The Complexity of Strong Orderability}

In this section, we consider the following decision problems.

STRONG $D I^{S}$-ORDERABILITY

Input: $\quad$ A set $X$ and a family of sets $\mathcal{X} \subseteq \mathcal{P}(X) \backslash\{\emptyset\}$.

Question: Is $\mathcal{X}$ strongly $D I^{S}$-orderable?

STRONG $D I$-ORDERABILITY

Input: $\quad$ A set $X$ and a family of sets $\mathcal{X} \subseteq \mathcal{P}(X) \backslash\{\emptyset\}$.

Question: Is $\mathcal{X}$ strongly $D I$-orderable?

We only present prove sketches due to space limitations. First, we proof that STRONG $D I^{S}$-ORDERABILITY is NPhard. This result will be superseded when we prove that the problem is $\Pi_{2}^{p}$-hard. However, it allows us to present in some detail the simplest form of a reduction that can be used with some modifications to prove Theorem 5, 6, 13 and 14.

Proposition 4. Strong $D I^{S}$-Orderability is NP-hard.

Proof (Sketch). Let $\phi$ be a instance of SAT with $n$ variables and $m$ clauses. We produce an instance $(X, \mathcal{X})$ of STRONG $D I^{S}$-ORDERABILITY. We produce this instance in a way that there is a linear order $\leq$ such that $\mathcal{X}$ is $D I^{S}$ orderable with respect to $\leq$ only if $\phi$ is satisfiable. Intuitively, we will code the truth value of a variable $V_{i}$ as a preference between two sets $X_{i}^{\mathrm{t}}$ and $X_{i}^{\mathrm{f}}$ by equating $X_{i}^{\mathrm{t}} \prec X_{i}^{\mathrm{f}}$ with $V_{i}$ is false and $X_{i}^{\mathrm{f}} \prec X_{i}^{\mathrm{t}}$ with $V_{i}$ is true. Then, for every clause, we add sets such that dominance and strict independence can not be jointly satisfied with respect to $\leq$ on these sets by any linear order that codes a truth assignment that does not satisfy the clause. This ensures that dominance and strict independence can only be satisfied with respect to $\leq$ by a linear order that codes a satisfying assignment of $\phi$.

First, we construct the set of elements $X$. For every variable $V_{i}$, the set $X$ contains elements $x_{i, 1}^{-}, x_{i, 2}^{-}, x_{i, 1}^{+}$and $x_{i, 2}^{+}$. Furthermore, it contains for every clause $C_{i}$ variables $z_{i}^{a}, y_{i}^{a}, \min _{i}^{a}$ and $\max _{i}^{a}$ for $a \in\{1,2,3\}$. Finally, it contains two elements $v_{1}$ and $v_{2}$. Then we define the following linear order $\leq$ on $X$ :

$$
\begin{gathered}
\min _{1}^{1}<\min _{1}^{2}<\cdots<\min _{m}^{3}<x_{1,1}^{-}<x_{1,2}^{-}<\ldots \\
<x_{n, 2}^{-}<v_{1}<v_{2}<z_{1}^{1}<z_{1}^{2} \cdots<z_{m}^{3}< \\
y_{1}^{1}<y_{1}^{2}<\cdots<y_{m}^{3}<x_{1,1}^{+}<x_{1,2}^{+}<\cdots \\
<x_{n, 2}^{+}<\max _{1}^{1}<\max _{1}^{2}<\cdots<\max _{m}^{3}
\end{gathered}
$$

Next, we construct the family $\mathcal{X}$. In the following, we write $Y:=\left\{x \in X \mid v_{1} \leq x \leq y_{3}^{m}\right\}$. First, we add for every variable $V_{i}$ sets $X_{i}^{\mathrm{t}}=Y \cup\left\{x_{i, 1}^{-}, x_{i, 1}^{+}\right\}$and $X_{i}^{\mathrm{f}}=Y \cup\left\{x_{i, 2}^{-}, x_{i, 2}^{+}\right\}$.

Now, let $C_{i}$ be a clause with variables $V_{j}, V_{k}, V_{l}$. We add

$$
\begin{aligned}
X_{j}^{\mathrm{t}} \backslash\left\{y_{i}^{1}\right\}, X_{j}^{\mathrm{f}} \backslash\left\{y_{i}^{1}\right\}, X_{k}^{\mathrm{t}} \backslash\left\{y_{i}^{2}\right\}, \\
X_{k}^{\mathrm{f}} \backslash\left\{y_{i}^{2}\right\}, X_{l}^{\mathrm{t}} \backslash\left\{y_{i}^{3}\right\} \text { and } X_{l}^{\mathrm{f}} \backslash\left\{y_{i}^{3}\right\} .
\end{aligned}
$$

By "reverse strict independence" 2 we know that the preference between $X_{j}^{\mathrm{t}} \backslash\left\{y_{i}^{1}\right\}$ and $X_{j}^{\mathrm{f}} \backslash\left\{y_{i}^{1}\right\}$ must be the same

\footnotetext{
${ }^{2}$ Every linear order satisfying strict independence has to satisfy reverse strict independence, i.e. $A \cup\{x\} \prec B \cup\{x\}$ implies $A \prec$ $B$ : Assume otherwise $B \prec A$ holds, then by strict independence $B \cup\{x\} \prec A \cup\{x\}$ must hold, contradicting $A \cup\{x\} \prec B \cup\{x\}$. Hence by the totality of $\preceq$ we have $A \prec B$.
} 
as the preference between $X_{j}^{\mathrm{t}}$ and $X_{j}^{\mathrm{f}}$. The same holds for the other two variables. Now, if all variables occur positively in $C_{i}$, we add sets such that $X_{j}^{\mathrm{f}} \backslash\left\{y_{i}^{1}\right\} \prec X_{k}^{\mathrm{t}} \backslash\left\{y_{i}^{2}\right\}$, $X_{k}^{\mathrm{f}} \backslash\left\{y_{i}^{2}\right\} \prec X_{l}^{\mathrm{t}} \backslash\left\{y_{i}^{3}\right\}$ and $X_{l}^{\mathrm{f}} \backslash\left\{y_{i}^{3}\right\} \prec X_{j}^{\mathrm{t}} \backslash\left\{y_{i}^{1}\right\}$ must hold in any order $\preceq$ on $\mathcal{X}$ that satisfies dominance and strict independence with respect to $\leq$. We call this enforcing these preferences. Then we get a contradiction if $V_{j}, V_{k}$ and $V_{l}$ are false because

$$
\begin{array}{r}
X_{j}^{\mathrm{t}} \backslash\left\{y_{i}^{1}\right\} \prec X_{j}^{\mathrm{f}} \backslash\left\{y_{i}^{1}\right\} \prec X_{k}^{\mathrm{t}} \backslash\left\{y_{i}^{2}\right\} \prec X_{k}^{\mathrm{f}} \backslash\left\{y_{i}^{2}\right\} \prec \\
X_{l}^{\mathrm{t}} \backslash\left\{y_{i}^{3}\right\} \prec X_{l}^{\mathrm{f}} \backslash\left\{y_{i}^{3}\right\} \prec X_{j}^{\mathrm{t}} \backslash\left\{y_{i}^{1}\right\}
\end{array}
$$

holds. If a variable, say $V_{j}$, occurs negatively in $C_{i}$, we switch $X_{j}^{\mathrm{t}}$ and $X_{j}^{\mathrm{f}}$ and enforce $X_{j}^{\mathrm{t}} \backslash\left\{y_{i}^{1}\right\} \prec X_{k}^{\mathrm{t}} \backslash\left\{y_{i}^{2}\right\}$ and $X_{l}^{\mathrm{f}} \backslash\left\{y_{i}^{3}\right\} \prec X_{j}^{\mathrm{f}} \backslash\left\{y_{i}^{1}\right\}$.

Next, we show how we can enforce these preference. Assume we want to enforce $X_{j}^{a} \backslash\left\{y_{i}^{1}\right\} \prec X_{k}^{b} \backslash\left\{y_{i}^{2}\right\}$ for $a, b \in$ $\{\mathrm{t}, \mathrm{f}\}$. We add $\left\{z_{i}^{1}\right\},\left\{z_{i}, \max _{i}^{1}\right\}$ and $\left(X_{j}^{a} \backslash\left\{y_{i}^{1}\right\}\right) \cup\left\{\max _{i}^{1}\right\}$. Our goal is to enforce $\left(X_{j}^{a} \backslash\left\{y_{i}^{1}\right\}\right) \cup\left\{\max _{i}^{1}\right\} \prec\left\{z_{i}^{1}, \max _{i}^{1}\right\}$ which forces by reverse strict independence $X_{j}^{a} \backslash\left\{y_{i}^{1}\right\} \prec$ $\left\{z_{i}^{1}\right\}$. Then we enforce $\left\{z_{i}^{1}\right\} \prec X_{k}^{b} \backslash\left\{y_{i}^{2}\right\}$ to get by transitivity $X_{j}^{a} \backslash\left\{y_{i}^{1}\right\} \prec X_{k}^{b} \backslash\left\{y_{i}^{2}\right\}$ as desired. To enforce $\left(X_{j}^{a} \backslash\right.$ $\left.\left\{y_{i}^{1}\right\}\right) \cup\left\{\max _{i}^{1}\right\} \prec\left\{z_{i}, \max _{i}^{1}\right\}$ we add a sequence of sets $A_{1}, A_{2}, \ldots, A_{l}$ such that $A_{1}=\left(X_{j}^{a} \backslash\left\{y_{i}^{1}, z_{i}^{1}\right)\right\} \cup\left\{\max _{i}^{1}\right\}$, $A_{i+1}=A_{i} \backslash \min _{\leq}\left(A_{i}\right)$ and $A_{l}=\left\{\max _{i}^{1}\right\}$. This enforces by dominance $A_{1} \prec A_{2} \prec \cdots \prec A_{l}$ which enforces by transitivity

$$
A_{1}=\left(X_{j}^{a} \backslash\left\{y_{i}^{1}, z_{i}^{1}\right\}\right) \cup\left\{\max _{i}^{1}\right\} \prec\left\{\max _{i}^{1}\right\}=A_{l} .
$$

Finally, this enforces by strict independence the desired $\left(X_{j}^{a} \backslash\left\{y_{i}^{1}\right\}\right) \cup\left\{\max _{i}^{1}\right\} \prec\left\{z_{i}, \max _{i}^{1}\right\}$. Using the same idea and $\min _{i}^{1}$ we enforce $\left\{z_{i}^{1}\right\} \prec X_{k}^{b} \backslash\left\{y_{i}^{2}\right\}$ finishing the construction for $X_{j}^{a} \backslash\left\{y_{i}^{1}\right\} \prec X_{k}^{b} \backslash\left\{y_{i}^{2}\right\}$. We enforce the other preferences for that clause $X_{k}^{c} \backslash\left\{y_{i}^{2}\right\} \prec X_{l}^{d} \backslash\left\{y_{i}^{3}\right\}$ and $X_{l}^{e} \backslash\left\{y_{i}^{3}\right\} \prec X_{j}^{a} \backslash\left\{y_{i}^{1}\right\}$ for $c, d, e \in\{\mathrm{t}, \mathrm{f}\}$ similarly using $z_{i}^{2}, \max _{i}^{2}$ and $\min _{i}^{2}$ resp. $z_{i}^{3}, \max _{i}^{3}$ and $\min _{i}^{3}$. We repeat this procedure for every clause. Now, by construction, $\mathcal{X}$ can only be $D I^{S}$-orderable with respect to $\leq$ if $\phi$ is a positive instance of SAT.

We omit how to construct a linear order $\preceq$ on $\mathcal{X}$ that satisfies dominance and strict independence with respect to $\leq^{\prime}$ for an arbitrary linear order $\leq^{\prime}$ on $X$ if $\phi$ is satisfiable.

We observe that this reduction also works as a reduction from SAT to $D I^{S}$-ORDERABILITY, as the constructed family is strongly $D I^{S}$-orderable if and only if it is $D I^{S}$ orderable with respect to a fixed linear order $\leq$. Next, we extend the construction to prove the $\Pi_{2}^{p}$-hardness of STRONG $D I^{S}$-ORDERABILITY.

Theorem 5. Strong $D I^{S}$-Orderability is $\Pi_{2}^{p}$ complete.

Proof (Sketch). $\Pi_{2}^{p}$-membership is clear as we can universally guess a linear order $\leq$ on $X$ and then check via the NPoracle if $\mathcal{X}$ is $D I^{S}$-orderable with respect to $\leq$. It remains to show that STRONG $D I^{S}$-ORDERABILITY is $\Pi_{2}^{p}$-hard. We do this by extending the reduction above to a reduction from a $\Pi_{2}$-SAT instance $\phi=\forall \vec{W} \exists \vec{V} \psi(\vec{W}, \vec{V})$. Let $w_{1} \ldots w_{l}$ be the universally quantified variables. We set up the reduction similarly to the one for Proposition 4. Additionally, we add for every universally quantified variable $w_{i}$ represented by $X_{i}^{\mathrm{t}}$ and $X_{i}^{\mathrm{f}}$ sets $X_{i}^{\mathrm{t}} \backslash\left\{y_{i}^{q}\right\}, X_{i}^{\mathrm{f}} \backslash\left\{y_{i}^{q}\right\},\left\{w_{i}^{\mathrm{t}}\right\},\left\{w_{i}^{\mathrm{f}}\right\}$ and $\left\{w_{i}^{\mathrm{t}}, w_{i}^{\mathrm{f}}\right\}$, where $y_{i}^{q}, w_{i}^{\mathrm{t}}$ and $w_{i}^{\mathrm{f}}$ are new elements. Then, we enforce - with the same method as above $-X_{i}^{\mathrm{t}} \backslash\left\{y_{i}^{q}\right\} \prec$ $\left\{w_{i}^{\mathrm{t}}\right\}$ and $\left\{w_{i}^{\mathrm{f}}\right\} \prec X_{i}^{\mathrm{f}} \backslash\left\{y_{i}^{q}\right\}$ using new elements $\min _{i}^{q}$ and $\max _{i}^{q}$. Now, let $\leq^{\prime}$ be a linear order on $X$ such that $w_{i}^{\mathrm{t}}<^{\prime} w_{i}^{\mathrm{f}}$ holds. Then $X_{i}^{\mathrm{t}} \prec X_{i}^{\mathrm{f}}$ must hold for every order $\preceq$ on $\mathcal{X}$ that satisfies dominance and strict independence with respect to $\leq '$. Analogously, we add sets and new elements such that $\bar{X}_{i}^{\mathrm{f}} \prec X_{i}^{\mathrm{t}}$ must hold for every order $\preceq$ on $\mathcal{X}$ that satisfies dominance and strict independence with respect to any linear order $\leq$ "l on $X$ such that $w_{i}^{\mathrm{f}}<^{\prime \prime} w_{i}^{\mathrm{t}}$ holds.

We claim that $(X, \mathcal{X})$ can only be a positive instance of Strong $D I^{S}$-ORdERABILITY if $\phi$ is satisfiable. First, we fix the same order $\leq$ as in the proof of Proposition 4 on the elements that occur already in that reduction. Then, for every truth assignment $T$ to the variables in $\vec{W}$ there is a linear order $\leq^{*}$ on $X$ that coincides with $\leq$ on the old elements such that $w_{i}^{\mathrm{t}}<^{*} w_{i}^{\mathrm{f}}$ if $w_{i}$ is assigned false in $T$ and $w_{i}^{\mathrm{f}}<^{*} w_{i}^{\mathrm{t}}$ if $w_{i}$ is assigned true in $T$. Now, if there is no satisfying assignment to $\phi$ that extends $T$, then there can be no order on $\mathcal{X}$ satisfying dominance and strict independence with respect to $\leq^{*}$. Hence $(X, \mathcal{X})$ can only be $D I^{S}$-orderable with respect to every linear order $\leq^{*}$ if $\phi$ is satisfiable.

We omit showing that if $\phi$ is satisfiable then $(X, \mathcal{X})$ is a positive instance of STRONG $D I^{S}$-ORDERABILITY.

It is also possible to extend the construction to prove the $\Pi_{2}^{p}$-completeness of STRONG $D I$-ORDERABILITY using an idea from Maly and Woltran (2017). We only give a brief description of the proof idea.

Theorem 6. Strong $D I$-ORderability is $\Pi_{2}^{p}$-complete.

Proof idea. We need to change the construction in two places compared to Theorem 5. First, to enforce a strict preference between the two sets $X_{i}^{a} \backslash\left\{y_{j}^{b}\right\}$ and $X_{k}^{c} \backslash\left\{y_{j}^{d}\right\}$ using independence instead of strict independence we can enforce $X_{i}^{a} \backslash\left\{y_{j}^{b}\right\} \preceq\left\{z_{j}^{e}\right\}$ and $\left\{\overline{z_{j}^{e}}\right\} \preceq X_{k}^{c} \backslash\left\{y_{j}^{d}\right\}$ following the construction above. Then, if we add $\left\{z_{j}^{e}, \overline{z_{j}^{e}}\right\}$ to $\mathcal{X}$ and set $z_{j}^{e}<\overline{z_{j}^{e}}$ this enforces $X_{i}^{a} \backslash\left\{y_{j}^{b}\right\} \prec X_{k}^{c} \backslash\left\{y_{j}^{d}\right\}$ by dominance. Second, we have to make sure that all preferences between all pairs of sets $X_{i}^{\mathrm{t}}$ and $X_{i}^{\mathrm{f}}$ are strict. We refer the reader to Maly and Woltran (2017) for a description how this can be achieved.

One important consequence of Theorem 5 and 6 is that, under some very mild complexity assumptions, it is not easy to lift an order on the objects to an order on a family of sets that satisfies dominance and (strict) independence even if the family is known to be strongly $D I^{(S)}$-orderable.

Corollary 7. Given a set $X$, a linear order $\leq$ on $X$ and $a$ strongly $D I$-orderable family of sets $\mathcal{X} \subseteq \mathcal{P}(X) \backslash\{\emptyset\}$. If coNP $\neq \Pi_{2}^{p}$, then there exists no polynomial time algorithm 
that produces on input $(X, \mathcal{X}, \leq)$ a weak order $\preceq$ on $\mathcal{X}$ that satisfies dominance and independence. The same holds if we replace independence by strict independence.

\section{Incomplete Preferences}

In this section, we investigate the effect of dropping the requirement that the lifted order should be total. It can be seen by carefully checking the proofs of Maly, Truszczyński, and Woltran (2018) that this has no effect for the special type of families considered in their paper if dominance and strict independence are considered. On the other hand, Maly and Woltran (2017) have shown that the order lifting problem becomes trivial if one only requires a preorder on the family of sets that satisfies dominance and independence.

Theorem 8 (Maly and Woltran 2017). For every set $X$, linear order $\leq$ on $X$ and family of sets $\mathcal{X} \subseteq \mathcal{P}(X) \backslash\{\emptyset\}$, there is a preorder that satisfies dominance and independence.

In other words, every family of sets is strongly $D I$ orderable if we only require the lifted order to be a preorder. However, most voting rules for incomplete preferences require partial orders as input (Xia and Conitzer 2011). If we require the ranking on $\mathcal{X}$ to be a partial order, then strict independence and independence coincide. Therefore, we will consider strongly partially $D I^{S}$-orderable families of sets, i.e. families of sets $\mathcal{X} \subseteq \mathcal{P}(X) \backslash\{\emptyset\}$ such that for every linear order $\leq$ on $X$ there exists a partial order on $\mathcal{X}$ that satisfies dominance and strict independence with respect to $\leq$. For that we recall a results from Maly and Woltran (2017).

Proposition 9 (Maly and Woltran 2017). Let be $X$ a set, $\leq$ a linear order on $X$ and $\mathcal{X} \subseteq \mathcal{P}(X) \backslash\{\emptyset\}$ a family of sets. If there is partial order on $\overline{\mathcal{X}}$ that satisfies dominance and strict independence then there is a unique subset-minimal partial order $\preceq_{\min }$ that satisfies dominance and strict independence with respect to $\leq$. Furthermore, $\preceq_{\min }$ can be computed in polynomial time.

This gives us a constructive polynomial time procedure for deciding partial $D I^{S}$-orderability. However, it turns out that it is still difficult to decide whether a given family of sets is strongly partially $D I^{S}$-orderable.

Strong Partial $D I^{S}$-ORderabiLITy

Input: $\quad$ A set $X$ and a family of sets $\mathcal{X} \subseteq \mathcal{P}(X) \backslash\{\emptyset\}$.

Question: Is $\mathcal{X}$ strongly partially $D I^{S}$-orderable?

Theorem 10. Strong Partial $D I^{S}$-Orderability is coNP-complete.

Proof (Sketch). Let $\phi$ be an instance of TAUT. We construct an instance $(S, \mathcal{X})$ of Strong Partial $D I^{S_{-}}$ ORDERABILITY. For every variable $X_{i}$ in $\phi$ we add new elements $x_{i}^{1}$ and $x_{i}^{2}$ to $S$. We call the set of these elements $X$. We will treat every order on $S$ as encoding a truth assignment by equating $x_{i}^{\mathrm{f}}<x_{i}^{\mathrm{t}}$ to $X_{i}$ is true and $x_{i}^{\mathrm{t}}<x_{i}^{\mathrm{f}}$ to $X_{i}$ is false. Then, we add for every disjunct new variables $y_{j}^{\mathrm{t}}, y_{j}^{\mathrm{f}}$. We call the set of these elements $Y$. Essentially, we want to add sets such that $\left\{y_{j}^{\mathrm{t}}\right\} \prec\left\{y_{j}^{\mathrm{f}}\right\}$ holds for the minimal partial order satisfying dominance strict independence with respect to $\leq$ if and only if $C_{j}$ is not satisfied by the truth assignment coded by $\leq$. Then we will add sets that lead to a contradiction if $\left\{y_{j}^{\mathrm{t}}\right\} \prec\left\{y_{j}^{\mathrm{f}}\right\}$ holds for all disjuncts.

To achieve this, we add for every disjunct $C_{j}$ elements $c_{j}$ as well as $d_{j}^{k}$ and $e_{j}^{k}$ for $k \leq 3$. Finally, we add new variables $u, v, z_{1}$ and $z_{2}$. In the following we call any linear order on $S$ that is derived by replacing $X$ with an arbitrary linear order on the elements in $X$ in the following linear order

$$
\begin{array}{r}
u<c_{1}<\cdots<c_{m}<y_{1}^{\mathrm{t}}<\cdots<y_{m}^{\mathrm{t}}< \\
d_{1}^{1}<\cdots<d_{m}^{3}<X<e_{1}^{1}<\cdots<e_{m}^{3}<y_{1}^{\mathrm{f}}<\cdots< \\
\quad y_{m}^{\mathrm{f}}<z_{1}<z_{2}<v
\end{array}
$$

a critical linear order. In the following, we write $\preceq_{\text {min }}$ for the minimal partial order satisfying dominance and strict independence with respect to some linear order on $S$.

Next, we build the family $\mathcal{X}$. We do this in a way such that $\mathcal{X}$ is not strongly $D I^{S}$-orderable if there is a non-satisfying assignment of $\phi$. First, we add singletons for all elements of $X$ and $Y$, and $\left\{x_{i}^{\mathrm{f}}, x_{i}^{\mathrm{t}}\right\}$ for all elements of $X$. Then, for every linear order $\leq$ we have $\left\{x_{i}^{\mathrm{f}}\right\} \prec_{\text {min }}\left\{x_{i}^{\mathrm{t}}\right\}$ if $x_{i}^{\mathrm{f}}<x_{i}^{\mathrm{t}}$ and, on the other hand, $\left\{x_{i}^{\mathrm{t}}\right\} \prec_{\min }\left\{x_{i}^{\mathrm{f}}\right\}$ if $x_{i}^{\mathrm{t}}<x_{i}^{\mathrm{f}}$

Next, we add sets such that there is a critical linear or$\operatorname{der} \leq$ on $S$ such that we have $\left\{y_{i}^{\mathrm{t}}\right\} \prec_{\min }\left\{y_{i}^{\mathrm{f}}\right\}$ for all $i \leq m$ if and only if $\phi$ is not a tautology. For every disjunct $C_{j}=X_{i_{1}} \wedge X_{i_{2}} \wedge X_{i_{3}}$ we add sets $\left\{y_{j}^{\mathrm{t}}, d_{j}^{k}\right\},\left\{y_{j}^{\mathrm{t}}, d_{j}^{k}, x_{i_{k}}^{\mathrm{f}}\right\}$, $\left\{d_{j}^{k}, x_{i_{k}}^{\mathrm{f}}\right\}$ for all $k \in\{1,2,3\}$ as well as

$$
\begin{aligned}
& \left\{x_{i_{k}}^{\mathrm{t}}, e_{j}^{k}\right\},\left\{x_{i_{k}}^{\mathrm{t}}, e_{j}^{k}, z_{1}\right\},\left\{x_{i_{k}}^{\mathrm{t}}, e_{j}^{k}, z_{1}, z_{2}\right\}, \\
& \quad\left\{e_{j}^{k}, z_{1}, z_{2}\right\},\left\{e_{j}^{k}, z_{1}, z_{2}, y_{f}^{j}\right\},\left\{z_{1}, z_{2}, y_{f}^{j}\right\},\left\{z_{2}, y_{f}^{j}\right\} .
\end{aligned}
$$

If any of the variables occurs negatively in $C_{j}$, we switch $x_{i_{k}}^{\mathrm{f}}$ and $x_{i_{k}}^{\mathrm{t}}$ in the construction. We claim that these sets ensure that $\left\{y_{j}^{\mathrm{t}}\right\} \prec_{\min }\left\{y_{j}^{\mathrm{f}}\right\}$ holds for any critical linear order whenever at least one literal in $C_{j}$ is false. We have

$$
\begin{aligned}
&\left\{y_{j}^{\mathrm{t}}\right\} \prec_{\text {min }}\left\{y_{j}^{1}, d_{j}^{k}\right\} \prec_{\text {min }} \\
&\left\{y_{j}^{\mathrm{t}}, d_{j}^{k}, x_{i_{k}}^{\mathrm{f}}\right\} \prec_{\text {min }}\left\{d_{j}^{k}, x_{i_{k}}^{\mathrm{f}}\right\} \prec_{\text {min }}\left\{x_{i_{k}}^{\mathrm{f}}\right\}
\end{aligned}
$$

by dominance and, hence, by transitivity $\left\{y_{j}^{\mathrm{t}}\right\} \prec_{\min }\left\{x_{i_{k}}^{\mathrm{f}}\right\}$. Similarly, we have $\left\{x_{i_{k}}^{\mathrm{t}}\right\} \prec_{\min }\left\{y_{j}^{\mathrm{f}}\right\}$. Hence, $\left\{x_{i_{k}}^{\mathrm{f}}\right\} \prec_{\text {min }}$ $\left\{x_{i_{k}}^{\mathrm{t}}\right\}$ implies $\left\{y_{j}^{\mathrm{t}}\right\} \prec_{\min }\left\{y_{j}^{\mathrm{f}}\right\}$ by transitivity.

Now, we add sets that lead to a contradiction if $\left\{y_{j}^{\mathrm{t}}\right\} \prec_{\min }$ $\left\{y_{j}^{\mathrm{f}}\right\}$ holds for all $j$. First we add $\{u\},\left\{u, c_{1}\right\},\left\{u, c_{1}, y_{1}^{\mathrm{t}}\right\}$, $\left\{u, c_{1}, y_{1}^{\mathrm{t}}, v\right\}$ and $\{u, v\}$. Then we know for any critical linear order by dominance and transitivity $\{u, v\} \prec_{\text {min }}$ $\left\{u, c_{1}, y_{1}^{\mathrm{t}}, v\right\}$. Similarly, we add sets such that

$$
\begin{aligned}
\left\{u, c_{1}, \ldots, c_{j}, y_{j}^{\mathrm{f}}, \ldots, y_{1}^{\mathrm{f}}, v\right\} \prec_{\min } & \\
& \left\{u, c_{1}, \ldots, c_{j+1}, y_{j+1}^{\mathrm{t}}, y_{j}^{\mathrm{f}}, \ldots, y_{1}^{\mathrm{f}}, v\right\}
\end{aligned}
$$

and $\left\{u, c_{1}, \ldots, c_{m}, y_{m}^{\mathrm{f}}, \ldots, y_{1}^{\mathrm{f}}, v\right\} \prec_{\min }\{u, v\}$ hold for any $j$ and critical linear order. Finally, we add sets such that

$$
\begin{aligned}
\left\{u, c_{1}, \ldots, c_{j}, y_{j}^{\mathrm{t}}, \ldots, y_{j-1}^{\mathrm{f}}, v\right\} \prec_{\min } & \\
& \left\{u, c_{1}, \ldots, c_{j}, y_{j}^{\mathrm{f}}, \ldots, y_{1}^{\mathrm{f}}, v\right\}
\end{aligned}
$$


holds for any linear order on $X$ if and only if $\left\{y_{j}^{\mathrm{t}}\right\} \prec_{\min }$ $\left\{y_{j}^{\mathrm{f}}\right\}$ holds for that linear order. Now it can be checked that for every critical linear order

$$
\begin{aligned}
& \{u, v\} \prec_{\min }\left\{u, c_{1}, y_{1}^{\mathrm{t}}, v\right\} \prec_{\text {min }} \\
& \left\{u, c_{1}, y_{1}^{\mathrm{f}}, v\right\} \prec_{\min }\left\{u, c_{1}, c_{2}, y_{2}^{\mathrm{t}}, y_{1}^{\mathrm{f}}, v\right\} \prec_{\min } \ldots \\
& \quad \prec_{\min }\left\{u, c_{1}, \ldots, c_{m}, y_{m}^{\mathrm{f}}, \ldots, y_{1}^{\mathrm{f}}, v\right\} \prec_{\min }\{u, v\}
\end{aligned}
$$

holds if $\left\{y_{j}^{\mathrm{t}}\right\} \prec_{\min }\left\{y_{j}^{\mathrm{f}}\right\}$ holds for all disjuncts, i.e. if the critical linear order codes an unsatisfying assignment. It follows that if $\phi$ is not a tautology, then $(X, \mathcal{X})$ is not strongly partial $D I^{S}$-orderable. We omit showing that $(X, \mathcal{X})$ is strongly partial $D I^{S}$-orderable if $\phi$ is a tautology.

\section{Succinct domain restrictions}

Finally, we turn our attention to succinctly represented families of sets. First, we will quickly review the basic results on succinctly represented problems from the literature and recall the definitions and lemmas we need. The study of succinct problems goes back to Galperin and Wigderson (1983) for graphs that are succinctly represented by a Boolean circuit. Later this approach was extended by Balcázar, Lozano, and Torán (1992) to arbitrary problems that are succinctly represented by boolean circuits in the following way.

Definition 11. We say a Boolean circuit $C_{w}$ with two output gates represents a binary string $w$ if for every input of a binary number $i$ the following holds:

- the first output is 1 if and only if $i \leq|w|$

- if the first output is 1 then the second output equals the $i$-th bit of $w$.

The succinct version $Q_{S}$ of a problem $Q$ is: Given a Boolean circuit $C_{w}$ representing a boolean string ${ }^{3} w$ decide whether $w \in Q$.

For example, SUCCINCT SAT - the succinct version of $\mathrm{SAT}$ - can be defined as follows:

SuCCINCT SAT

Input: $\quad$ A Boolean circuit $C_{w}$ representing a word $w$. Question: Is the 3-CNF represented by $w$ satisfiable?

SuCCINCT SAT is known to be NEXP-complete (Papadimitriou 1994). Hence, SUCCINCT TAUT is coNEXPcomplete. Succinct versions of the problems considered in this paper be can defined similarly. The main tool to determine the complexity of succinct problems are so-called Conversion Lemmas. We use the Conversion Lemma by Balcázar, Lozano, and Torán (1992). Stronger versions of this lemma exist, for example by Veith (1998). However, the Conversion Lemma of Balcázar, Lozano, and Torán (1992) suffices for our purposes and has the advantage that only comparably simple reductions are used, namely ptime reductions and polylogtime reductions. Polylogtime reductions are reductions that - given random access to the input

\footnotetext{
${ }^{3}$ It is not important what specific encoding is used as long as the number of variables and clauses as well as the i-th variable in the $\mathrm{j}$-th clause can be read in polylog time. Any reasonable encoding will satisfy this requirement.
}

- need only $O\left(\log ^{c}(n)\right)$-time to output an arbitrary bit of the output. For a formal definition see for example (Murray and Williams 2017).

Lemma 12 (Conversion Lemma). Let $Q$ and $Q^{*}$ be decision problems. If $Q \leq$ polylogtime $Q^{*}$ then $Q_{S} \leq$ ptime $Q_{S}^{*}$.

We recall that the reduction used to prove that STRONG $D I^{S}$-ORDERABILITY is NP-hard also reproves that $D I^{S}$ ORDERABILITY is NP-hard. Therefore, we can use the Conversion Lemma to prove the following theorem.

Theorem 13. SUCCINCT $D I^{S}$-ORDERABILITY is NEXPcomplete. SUCCINCT STRONG $D I^{S}$-ORDERABILITY and SUCCINCT STRONG DI-ORDERABILITY are NEXP-hard.

Proof (Sketch). SuCCINCT $D I^{S}$-ORdERABILITY can be solved in NEXP-time by explicitly computing the family $\mathcal{X}$ and then solving the (exponentially larger) explicit problem in NP-time. For the hardness, we only have to check that the reduction presented in this paper is computable in polylogtime. We observe that the number and size of the sets in the family $\mathcal{X}$ only depends on the number of variables and not on the structure of the formula $\phi$. Using this observation, it is possible to prove that for a suitable encoding of $(X, \mathcal{X})$ resp. $(X, \mathcal{X}, \leq)$ the value of a bit can be decided by looking at one clause and which clause again only depends on the number of variables. Determining the number of variables and clauses and reading one clause can be done in polylogtime for any reasonable encoding of SAT. A similar argument works for strong $D I$-orderability.

Moreover, we note that the Conversion Lemma can also be applied to the reduction from TAUT to STRONG PARTIAL $D I^{S}$-ORDERABILITY.

Theorem 14. SUCCINCT STRONG PARTIAL $D I^{S}$ ORDERABILITY is coNEXP-complete.

\section{The extension rule}

Finally, we observe that adding the extension rule indeed has no influence on the complexity of the discussed problems.

Remark 15. It can be checked in polynomial time if an order satisfies the extension rule. Therefore, all arguments about containment in a complexity class stay valid. Furthermore, all reductions presented in this paper also work, with minimal changes, if the extension rule is additionally required. In the reductions for Proposition 4 and Theorem 5, 6 and 13 it is possible to construct an order that satisfies dominance, (strict) independence and the extension rule whenever is possible to construct an order that satisfies dominance and (strict) independence. The reduction for Theorem 10 and 14 needs to be changed slightly if the extension rule is required. Namely, every occurrence of $y_{k}^{\mathrm{t}}$ and $y_{k}^{\mathrm{f}}$ must be replaced by two elements $y_{k}^{\mathrm{t}}$ and $y_{k}^{\mathrm{t}^{*}}$ resp. $y_{k}^{\mathrm{f}}$ and $y_{k}^{\mathrm{f}^{*}}$ i.e., the truth value of disjunct $k$ is coded by the preference between $\left\{y_{k}^{\mathrm{t}}, y_{k}^{\mathrm{t}^{*}}\right\}$ and $\left\{y_{k}^{\mathrm{f}}, y_{k}^{\mathrm{f}^{*}}\right\}$.

\section{Discussion}

Our results show that we cannot easily recognize families of sets that are strongly $D I$ resp. $D I^{S}$-orderable. Furthermore, 
they show that it is hard to compute a total order satisfying dominance and (strict) independence even on strongly $D I$ or $D I^{S}$-orderable families. This limits the usefulness of the order lifting approach to combinatorial voting as voters may want the ability to easily reproduce the lifting process. However, this does not hold true if one only requires the lifted order to be partial. The coNP-hardness of STRONG PARTIAL $D I$-ORDERABILITY is not as problematic if the family of sets is of tractable size. Determining if the family of sets is strongly orderable is important but often not timesensitive. Therefore, we believe that lifting orders on candidates to partial orders on committees may be a promising approach to deal with the problems of combinatorial voting. However, future work is needed on the interplay between lifting procedures and voting rules, as well as on axioms besides dominance and (strict) independence.

Unfortunately, in many applications, the set of possible winning committees is too large to handle directly, making a succinct representation necessary. Our results show that this can lead to a rise in complexity that may make recognizing strongly orderable families intractable even in applications that are not time-critical. On the other hand, Maly, Truszczyński, and Woltran (2018) showed that less expressive succinct representations can avoid this problem. Unfortunately, the representation considered in their paper is very limiting. Therefore, future research is needed to identify succinct representations that are as expressive as possible without an exponential blow up in complexity,

\section{Acknowledgments}

This work was funded by the Austrian Science Fund (FWF) under grant number Y698 and P31890.

\section{References}

Balcázar, J. L.; Lozano, A.; and Torán, J. 1992. The complexity of algorithmic problems on succinct instances. In Computer Science: Research and Applications. Springer US. 351-377.

Barberà, S., and Pattanaik, P. K. 1984. Extending an order on a set to the power set: some remarks on Kannai and Peleg's approach. Journal of Economic Theory 32(1):185-191.

Barberà, S.; Bossert, W.; and Pattanaik, P. K. 2004. Ranking sets of objects. In Handbook of Utility Theory. Springer. 893-977.

Bossert, W., and Suzumura, K. 2012. Revealed preference and choice under uncertainty. SERIEs: Journal of the Spanish Economic Association 3(1):247-258.

Bossert, W.; Pattanaik, P. K.; and Xu, Y. 2000. Choice under complete uncertainty: Axiomatic characterizations of some decision rules. Economic Theory 16(2):295-312.

Boutilier, C., and Rosenschein, J. S. 2016. Incomplete information and communication in voting. In Handbook of computational social choice. Cambridge University Press. 223-257.

Bouveret, S.; Chevaleyre, Y.; and Maudet, N. 2016. Fair allocation of indivisible goods. In Handbook of Computational Social Choice. Cambridge University Press. 284-310.
Brandt, F., and Brill, M. 2011. Necessary and sufficient conditions for the strategyproofness of irresolute social choice functions. In Proc. TARK, 136-142. ACM.

Brandt, F.; Saile, C.; and Stricker, C. 2018. Voting with ties: Strong impossibilities via SAT solving. In Proc. AAMAS, 1285-1293. ACM.

Can, B.; Erdamar, B.; and Sanver, R. 2009. Expected utility consistent extensions of preferences. Theory and Decision 67(2):123-144.

Galperin, H., and Wigderson, A. 1983. Succinct representations of graphs. Information and Control 56(3):183 - 198.

Geist, C., and Endriss, U. 2011. Automated search for impossibility theorems in social choice theory: Ranking sets of objects. J. Artif. Intell. Res. (JAIR) 40:143-174.

Kannai, Y., and Peleg, B. 1984. A note on the extension of an order on a set to the power set. Journal of Economic Theory 32(1):172-175.

Kilgour, D. M. 2016. Approval elections with a variable number of winners. Theory and Decision 81.

Kraft, C. H.; Pratt, J. W.; and Seidenberg, A. 1959. Intuitive probability on finite sets. The Annals of Mathematical Statistics 30(2):408-419.

Lang, J., and Xia, L. 2016. Voting in combinatorial domains. In Handbook of computational social choice. Cambridge University Press. 197-221.

Larbi, R. B.; Konieczny, S.; and Marquis, P. 2010. A characterization of optimality criteria for decision making under complete ignorance. In Proc. KR'10, 172-181. AAAI Press. Maly, J., and Woltran, S. 2017. Ranking specific sets of objects. Datenbank-Spektrum 17(3):255-265.

Maly, J.; Truszczyński, M.; and Woltran, S. 2018. Preference orders on families of sets - When can impossibility results be avoided? In Proc. IJCAI, 433-439. IJCAI Organization.

Moretti, S., and Tsoukiàs, A. 2012. Ranking sets of possibly interacting objects using Shapley extensions. In Proc. KR, 199-209. AAAI Press.

Murray, C. D., and Williams, R. R. 2017. On the (non) NPhardness of computing circuit complexity. Theory of Computing 13(4):1-22.

Papadimitriou, C. H. 1994. Computational Complexity. Addison-Wesley.

Pattanaik, P. K., and Xu, Y. 1990. On ranking opportunity sets in terms of freedom of choice. Recherches Économiques de Louvain/Louvain Economic Review 56(3-4):383-390.

Terzopoulou, Z., and Endriss, U. 2019. Aggregating incomplete pairwise preferences by weight. In Proc. IJCAI, 595-601. IJCAI Organization.

Veith, H. 1998. Succinct representation, leaf languages, and projection reductions. Information and Computation 142(2):207-236.

Xia, L., and Conitzer, V. 2011. A maximum likelihood approach towards aggregating partial orders. In Proc. IJCAI, 446-451. AAAI Press. 